\title{
Efecto de los Campos Cristalinos en un Ferromagneto de Ising Mixto Bidimensional
}

\author{
Nicolás A. De La Espriella, Gladys R. Casiano y César Ortega \\ Universidad de Córdoba, Departamento de Física y Electrónica, Grupo Gamasco, Facultad de \\ Ciencias Básicas, Carrera 6 No. 76-103, Córdoba-Colombia. (e-mail: ndelae52@gmail.com)
}

Recibido Feb. 17, 2012; Aceptado Mar. 27, 2012; Versión final recibida Abr. 22, 2012

\section{Resumen}

Mediante simulaciones de Monte Carlo, se estudia el comportamiento magnético de un sistema de Ising ferromagnético, sobre una red cuadrada compuesta por dos subredes $\mathrm{A}$ y $\mathrm{B}$, con espines $S_{i}^{A}$ $=3 / 2$, y $\sigma_{j}^{B}=5 / 2$, y campos cristalinos $D_{A}$ y $D_{B}$, respectivamente. La interacción de intercambio $J_{1}$, entre espines $S_{i}^{A}$ y $\sigma_{j}^{B}$, es ferromagnética. Se calcula el diagrama de estados base, con diversos puntos donde coexisten tres fases. Se analiza el efecto de los campos sobre las magnetizaciones de las subredes, el calor específico y la temperatura crítica. Se hallan posibles transiciones de primer orden para $D_{A}=D_{B}, D_{A}<0$ y $D_{B}<0$. Cuando alguno de los campos es nulo y el otro se incrementa, la temperatura crítica se incrementa hasta un valor límite. El efecto de $D_{B}>0$ es más determinante en el ordenamiento del sistema, que el efecto de $D_{A}>0$.

\section{Crystal Field Effects in a Mixed Two Dimensional Ising Ferromagnet}

\begin{abstract}
Through Monte Carlo simulations, the magnetic behavior of Ising ferromagnetic system on a square lattice composed of two sublattices $A$ and $B$, with spins $S_{i}^{A}=3 / 2$ y $\sigma_{i}^{B}=5 / 2$, and crystalline fields $D_{A}$ and $D_{B}$, are studied. The exchange interaction $J_{1}$, between spins $S_{i}^{A}$ and $\sigma_{i}^{B}$, is ferromagnetic. The diagram of ground states, with different points where three phases coexist was calculated. The effect of the fields on the magnetization of the sublattices, specific heat and critical temperature was analyzed. Possible first-order phase transitions are found for $D_{A}=D_{B}, D_{A}<0$ and $D_{B}<0$. When any of the fields is zero and the other increases, the critical temperature increases to a threshold value. The effect of $D_{B}>0$ is more crucial in the order of the system, that the effect de $D_{A}>0$.
\end{abstract}

Keywords: mixed Ising model, Monte Carlo, critical temperatures, crystal fields 


\section{INTRODUCCIÓN}

Aunque el magnetismo en materia condensada ha atraído la atención de muchos investigadores en el campo de las ciencias físicas, su comprensión es todavía una tarea difícil que requiere elementos de los diversos campos de la física actual. Por ejemplo, la comprensión de la anisotropía magnética en los magnetos moleculares, la cual es de gran importancia para muchas aplicaciones tecnológicas.

La caracterización de las propiedades termodinámicas de los materiales magnéticos moleculares, requiere sofisticados métodos de la física estadística y del magnetismo molecular. Este último se considera un área interdisciplinaria donde se explotan las técnicas de la química molecular, con el propósito de diseñar y sintetizar nuevos materiales magnéticos basados en redes moleculares, en lugar de las redes continuas de los magnetos clásicos (Gatteschi y Sessoli, 2004). El magnetismo de superficies ha tomado un gran auge, debido a sus relevantes aplicaciones tecnológicas. Por ejemplo, los sustratos antiferromagnéticos son importantes en los sensores magnéticos, ya que aseguran el endurecimiento magnético de una de las capas ferromagnéticas del sensor. El estudio de este tipo de propiedades está enfocado hacia las aplicaciones en la espintrónica (Belmamount et al, 2009).

El interés por la investigación, tanto teórica como experimental, de los materiales magnéticos moleculares, ha crecido notablemente en las últimas dos décadas, debido a que pueden mostrar, por un lado, histéresis magnética en todo el volumen, y por el otro, presentar importantes efectos cuánticos (Keskin et al., 2009; Dely et al., 2009; Gatteschi y Sessoli, 2004). Por otra parte, comparados con los metales clásicos y los óxido-metales, los magnetos moleculares tienen la ventaja de que se pueden obtener a través de una selección apropiada de fuentes de espín, tales como iones en los metales de transición y radicales orgánicos (Ohkoshi y Hashimoto, 2002, Luo et al., 2009). También poseen múltiples aplicaciones tecnológicas, las cuales se sustentan en fenómenos tales como el ordenamiento magnético a temperatura ambiente, momentos magnéticos espontáneos, alta coercitividad (Blundell y Pratt, 2004) y la aparición de temperaturas de compensación (Mohamad, 2011). Esta última propiedad, asociada con el incremento de la coercitividad, permite la formación de pequeños dominios magnéticos estables, situación que se puede aplicar en la escritura y borrado de memorias magneto-ópticas (Hurtado y Buendía, 2003).

La simulación y el estudio numérico, mediante modelos de Ising de espines mixtos, de diversos magnetos moleculares, se han convertido en una importante herramienta teórica para su análisis y comprensión (Deviren et al., 2009). Aunque inicialmente los sistemas de Ising de espines mixtos se estudiaron principalmente por curiosidad teórica, en la actualidad se plantean como un excelente "laboratorio" para la comprensión de las propiedades termomagnéticas de algunos materiales moleculares magnéticos bidimensionales y tridimensionales (Nakamura y Tucker, 2002; Keskin y Polat, 2009). Recientemente se ha dedicado un gran esfuerzo al estudio de los fenómenos críticos en los sistemas de Ising de espines mixtos, ya que son de gran interés para la caracterización de cierto tipo de ferrimagnetismo que se presenta en diversos materiales moleculares magnéticos (Deviren et al., 2011; Deviren et al., 2010; Keskin et al., 2009). Por ejemplo, los compuestos cristalinos no-metálicos o intermetálicos con $A_{p} B_{1-p}$, donde $A$ y $B$ son átomos magnéticos distintos, son ferrimagnetos. Estos ferrimagnetos se han analizado ampliamente por sus aplicaciones tecnológicas (Mohamad, 2011). No sólo se han estudiado sistemas ferrimagnéticos, sino que también se han caracterizado las propiedades magnéticas de sistemas ferromagnéticos orgánicos (Nakamura y Tucker, 2002), los cuales se han sintetizado satisfactoriamente, aunque sigue abierta la posibilidad de seguir analizando el mecanismo del ferromagnetismo en estos materiales (Luo et al., 2009).

Otros sistemas de interesante comportamiento magnético, que se han estudiado por medio de modelos de Ising de espines mixtos, son las nanopartículas magnéticas. Modelos muy útiles en las aplicaciones industriales de las emergentes nanotecnologías, que se pueden usar para una variedad de nano-dispositivos, debido a su reducido tamaño e importantes propiedades magnéticas, tales como memorias magnéticas de alta densidad, sensores y dispositivos de imagen molecular, entre otros (Zaim y Kerouad., 2010; Zaim et al., 2009a). De igual forma, 
algunos de estos modelos de Ising mixto, como los de interacciones multiespín (Zaim et al., 2009b) y campo cristalino aleatorio se pueden usar, desde el punto de vista experimental, para describir diversos sistemas físicos como fluidos clásicos, ${ }^{3} \mathrm{H}_{\mathrm{e}}$ sólido, bicapas lípidas y gases raros (Benayad y Ghliyem, 2012).

Otra utilidad destacada de los modelos de Ising de espines mixtos, es la simulación de los materiales análogos al azul de Prusia. Estos materiales son una clase especial de magnetos moleculares, interesantes por la relativa facilidad de controlar sus propiedades durante los procesos de síntesis, mediante la selección deseada de fuentes de espín (Buendía y Villarroel, 2007; Ohkoshi y Hashimoto, 2002; Dely et al., 2009). Los recientes estudios del comportamiento crítico y de compensación de una aleación ternaria de espines mixtos del tipo $A B_{p} C_{1-p}$, sobre una red cúbica, enfocada sobre el caso de los parámetros correspondientes al análogo del azul de Prusia $\left(\mathrm{Ni}_{\mathrm{p}}{ }^{\mathrm{I}} \mathrm{Mn}_{1-\mathrm{p}}{ }^{\prime \prime}\right)_{1.5}\left[\mathrm{Cr}{ }^{\prime \prime \prime}(\mathrm{CN})_{6}\right] . n \mathrm{H}_{2} \mathrm{O}$ (Zukovic y Bobak, 2010), así lo demuestran.

En este trabajo desarrollamos simulaciones de Monte Carlo, técnica confiable en la solución de modelos de Ising mixto (Selke y Oitmaa, 2010; De La Espriella et al., 2012a; De La Espriella et al., 2012b;), para investigar las propiedades magnéticas de un modelo ferromagnético de Ising mixto de espines $S_{i}^{A}=3 / 2$ y $\sigma_{j}^{B}=5 / 2$, con distintos campos cristalinos. El modelo se ha estudiado por diversos métodos, entre los que se destacan las relaciones de recursión exacta (Yessoufou et al., 2009; Albayrak e Yigit, 2007), teoría de campo medio (Mohamad, 2011; Deviren y Keskin, 2010), Monte Carlo (De La Espriella y Buendía, 2011; De La Espriella y Buendía, 2010, De La Espriella et al., 2012a), teoría de campo efectivo (Zhang et al., 2005). En cada una de estas investigaciones se establece que el sistema presenta una rica variedad de fenómenos multicríticos, entre los que se destacan transiciones de fase de primer y segundo orden, temperaturas de compensación y comportamientos reentrantes.

\section{MODELO Y SIMULACIONES}

El modelo estudiado es un ferromagneto de Ising mixto de espines $3 / 2$ y $5 / 2$, alternados sobre una red cuadrada de lado $L=80$. El Hamiltoniano de interacción del sistema se define como:

$$
H=-J_{1} \sum_{<i j>} S_{i}^{A} \sigma_{j}^{B}+D_{A} \sum_{i}\left(S_{i}^{A}\right)^{2}+D_{B} \sum_{j}\left(\sigma_{j}{ }^{B}\right)^{2}
$$

donde $S_{i}^{A}= \pm 3 / 2, \pm 1 / 2$ y $\sigma_{j}^{B}= \pm 5 / 2, \pm 3 / 2, \pm 1 / 2$, son los espines sobre las subredes $A$ y $B$ respectivamente. $J_{1}$ representa la interacción de intercambio entre pares de espines a primeros vecinos, $D_{A}$ y $D_{B}$ son los campos cristalinos de cada subred, generadores de las anisotropías del sistema. La primera suma se efectúa sobre todos los pares de espines con interacción a primeros vecinos, es decir, entre los sitios con espines $S_{i}^{A}=3 / 2$ y $\sigma_{j}^{B}=5 / 2$, y las sumas $\Sigma_{i}$ y $\Sigma_{j}$ se realizan sobre todos los sitios de espines de las subredes $A$ y $B$, respectivamente. Escogemos un acoplamiento ferromagnético a primeros vecinos, $J_{1}>0$, y adoptamos condiciones de borde periódicas. Todas las variables en el Hamiltoniano están en unidades de energía.

La simulación del modelo, descrito por la ecuación (1), se lleva a cabo mediante el método de Monte Carlo, generando los estados aleatoriamente mediante un algoritmo tipo baño térmico. Se elige un espín al azar, y se calcula la diferencia de energía $\Delta \mathrm{E}_{\mathrm{ij}}$ y la probabilidad de transición $\exp \left(-\beta \Delta \mathrm{E}_{\mathrm{ij}}\right)$, asociada a cada uno de los posibles cambios. Luego, se considera si el espín cambia su valor, generando un número aleatorio $\theta$, en el intervalo $\left(0, \Sigma \mathrm{P}_{\mathrm{i}}\right)$, donde $\Sigma \mathrm{P}_{\mathrm{i}}$, representa la suma de las probabilidades de transición. Los datos se generan con $5 \times 10^{4}$ pasos de Monte Carlo por sitio, después de descartar los primeros $10^{4}$ pasos por sitio para alcanzar el equilibrio del sistema. El cálculo de errores se estima usando el método de bloques, donde se divide la muestra de tamaño $L$ en $n_{b}$ bloques de longitud $L_{b}=L / n_{b}$. Cuando $L_{b}$ es mayor que la longitud de correlación, los promedios de los bloques se pueden considerar estadísticamente independientes. De esta forma, los errores se calculan tomando los promedios de los bloques en vez de las mediciones originales. Las barras de error se calculan agrupando todas las medidas en 10 bloques y tomando su desviación estándar (Newman y Barkema, 2006; De La Espriella et al., 2012a). 
Las magnetizaciones por sitio de las subredes $\left(M_{A}, M_{B}\right)$, y la magnetización total por espín, $M_{T}$, se definen como:

$$
M_{A}=\frac{2}{L^{2}}\left(<\sum_{i} S_{i}{ }^{A}>\right) \quad M_{B}=\frac{2}{L^{2}}\left(<\sum_{j} \sigma_{j}{ }^{B}>\right) \quad M_{T}=\frac{M_{A}+M_{B}}{2}
$$

Definiendo $\beta=1 / k_{B} T$, se calcula el calor específico por sitio, $C$, mediante la expresión:

$$
C=\frac{\beta^{2}}{L^{2}}\left(<H^{2}>-<H>^{2}\right)
$$

\section{RESULTADOS NUMÉRICOS Y DISCUSIÓN}

Los resultados inician calculando el diagrama de fase de estados base del modelo en forma exacta, en el plano $\left(D_{B} /\left|J_{1}\right|, D_{A} /\left|J_{1}\right|\right)$, como se nota en la figura 1 . En la tabla 1 se muestran las energías, calculadas numéricamente, cuando $J_{1}>0$, asociadas a las configuraciones de espín $2 \times 2$ de estados base, dos espines tipo $S_{i}^{A}$ y dos $\sigma_{j}^{B}$. El conocimiento de los diagramas de fase a $\mathrm{T}=0$ es muy instructivo para la comprensión de los resultados de los diagramas de fase a temperatura finita, dado que son relevantes para identificar regiones donde el sistema pudiera presentar comportamientos magnéticos importantes.

Tabla 1: Energías de estados base del modelo $J_{1}-D_{A}-D_{B}$.

\begin{tabular}{|cc|c|c|}
\hline Estado base: $\left|\begin{array}{cc}S_{i}^{A} & \sigma_{j}^{B} \\
\sigma_{j}^{B} & s_{i}^{A}\end{array}\right|$ & Energía & Región \\
\hline$\mp 3 / 2$ & $\mp 5 / 2$ & $-30 J_{1}-4.5 D_{A}-12.5 D_{B}$ & 1 \\
$\mp 5 / 2$ & $\mp 3 / 2$ & & \\
\hline$\mp 3 / 2$ & $\mp 3 / 2$ & $-18 J_{1}-4.5 D_{A}-4.5 D_{B}$ & 2 \\
$\mp 3 / 2$ & $\mp 3 / 2$ & & 3 \\
\hline$\mp 3 / 2$ & $\mp 1 / 2$ & $-6 J_{1}-4.5 D_{A}-0.5 D_{B}$ & 3 \\
$\mp 1 / 2$ & $\mp 3 / 2$ & & 4 \\
\hline $1 / 2$ & $\mp 1 / 2$ & $-2 J_{1}-0.5 D_{A}-0.5 D_{B}$ & 4 \\
\hline$\mp 1 / 2$ & $\mp 1 / 2$ & & \\
\hline$\mp 1 / 2$ & $\mp 3 / 2$ & $-6 J_{1}-0.5 D_{A}-4.5 D_{B}$ & 5 \\
\hline $3 / 2$ & $\mp 1 / 2$ & & \\
\hline $1 / 2$ & $\mp 5 / 2$ & $-10 J_{1}-0.5 D_{A}-12.5 D_{B}$ & 6 \\
\hline $5 / 2$ & $\mp 1 / 2$ & & \\
\hline
\end{tabular}

El diagrama de fase de estados base, en la figura 1, muestra la existencia de cuatro puntos donde coexisten tres fases: $P_{1}, P_{2}, P_{3}$ y $P_{4}$. En la tabla 1 , se aprecia que los cambios de fase presentados en las regiones 1,2 y 3 , ocurren debido al cambio de los momentos en la subred de espines $\sigma_{j}^{B}$. Las configuraciones de estados base en las fases 3 y 4 , nos indican que, para valores grandes del módulo de campo cristalino $\left|D_{B}\right|$, cuando $D_{B}<0$, la subred B está formada sólo por espines $\mp 1 / 2$. También se nota que la magnetización total del sistema disminuye gradualmente desde la región 1 hasta la región 4. 


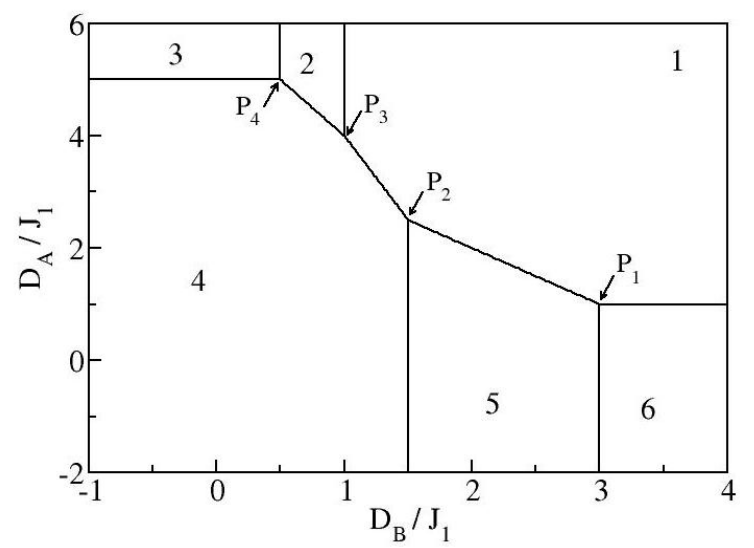

Fig. 1: Diagrama de fase a $T=O K$ para el modelo $J_{1}-D_{A}-D_{B}$.

\section{Efecto de los campos cristalinos $D_{A}$ y $D_{B}$}

Se analiza el efecto de los campos cristalinos $D_{A}^{\prime}=D_{A} / J_{1}$ y $D_{B}^{\prime}=D_{B} / J_{1}$, sobre las magnetizaciones de las subredes, el calor específico por espín y la temperatura crítica del sistema. En las figuras 2(a)-(b) se exhibe el comportamiento de las magnetizaciones de las subredes como funciones de la temperatura, cuando $D_{A}^{\prime}=0$ y $D_{B}^{\prime} \geq 0$. Los resultados de las magnetizaciones en $T=0$, coinciden con los reportados en el diagrama de fase de estados base de la figura 1, y la tabla 1.
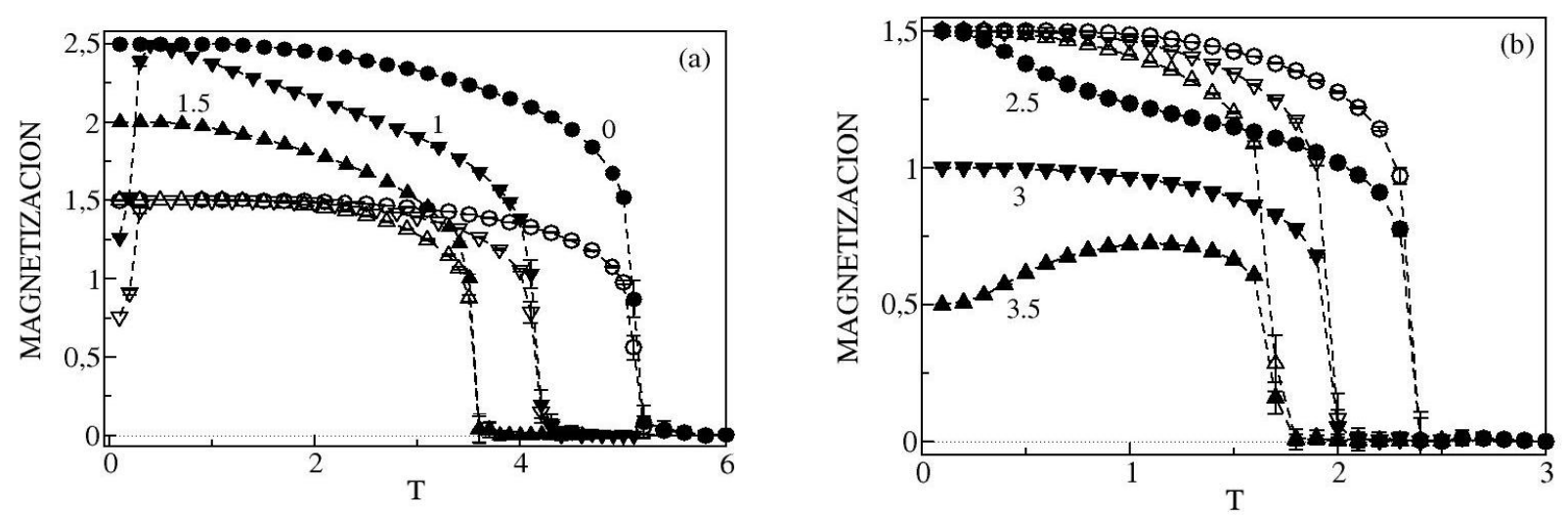

Fig. 2: Comportamiento de las magnetizaciones de las subredes, $\left|M_{A}(T)\right|$ (símbolos vacíos) y $\left|\mathrm{M}_{\mathrm{B}}(\mathrm{T})\right|$ (símbolos llenos). En (a) y (b) $D_{A}^{\prime}=0$, y los valores de $D_{B}^{\prime}$ se muestran en las curvas. Los valores de $D_{B}^{\prime}$ para $\left|\mathrm{M}_{A}(\mathrm{~T})\right|$ son iguales a los de $\left|\mathrm{M}_{B}(\mathrm{~T})\right|$, para el símbolo correspondiente.

El comportamiento de las magnetizaciones de las subredes en las figuras 2(a)-(b), nos muestra que la temperatura crítica es una función decreciente del campo cristalino $D_{B}^{\prime}$. Además, $\left|\mathrm{M}_{A}(T)\right|$ tiene un único valor de saturación (1.5) en $T=0$, en tanto que $\left|\mathrm{M}_{B}(T)\right|$ presenta cinco valores.

En la figura 2(a), se presenta un fenómeno interesante cuando $D_{B}^{\prime}=1$. Se observa, a bajas temperaturas, una posible transición de fase de primer orden. Las magnetizaciones de las subredes del sistema saltan abruptamente desde $\left|\mathrm{M}_{A}(T)\right| \approx 0.75$ y $\left|\mathrm{M}_{B}(T)\right| \approx 1.25$, hasta alcanzar sus valores de saturación en $\left|M_{A}(T)\right|=1.5$ y $\left|M_{B}(T)\right|=2.5$. Para los demás casos, de la figura 2(a), las magnetizaciones caen continuamente desde sus valores de saturación en $T=0$, hasta alcanzar una transición de segundo orden en $T=T_{C}$. A medida que se incrementa la temperatura, las magnetizaciones van decreciendo hasta hacerse cero en $T_{C}$, el punto donde el sistema hace la transición a la fase desordenada o paramagnética. Cuando $D_{A}^{\prime}=D_{B}^{\prime}=0$, no se considera influencia de los campos cristalinos sobre el sistema, y tenemos el "modelo $J_{1}$ ", el típico comportamiento de un ferromagneto, donde el sistema sólo experimenta la interacción de intercambio a primeros vecinos en la red. 
En la figura 2(b), se observa que la subred de espines $S_{i}^{A}$ se mantiene más ordenada que la subred $B$, debido a que no se siente afectada por la acción del campo $D^{\prime}{ }_{A}$. Las curvas de $\left|M_{A}(T)\right|$ se asemejan a las de un sistema ferromagnético. También se observa que a medida que se incrementa el campo $D_{B}^{\prime}$ los valores de $\left|\mathrm{M}_{B}(T=0)\right|$ decrecen.

El modelo resulta más interesante cuando los campos cristalinos $D_{A}^{\prime}$ y $D_{B}^{\prime}$ son diferentes de cero. En la gráfica 3(a) se analiza el caso cuando las intensidades de los campos de anisotropía de ión simple tienen la misma magnitud y son distintos de cero. Se describe el comportamiento de las magnetizaciones de las subredes, para $D_{A}^{\prime}=D_{B}^{\prime}=1.0, D_{A}^{\prime}=D_{B}^{\prime}=1.5$ y $D_{A}^{\prime}=D_{B}^{\prime}=1.7$. De las variaciones termo-magnéticas presentadas en la figura $3(a)$, se observa que, en virtud del incremento de los valores de los campos cristalinos, la temperatura crítica decrece.

Para los valores $D_{A}^{\prime}=D_{B}^{\prime}=1,1.5$ de la figura 3(a), el comportamiento de las magnetizaciones de las subredes solamente manifiesta transiciones de fase continuas 0 de segundo orden, donde las temperaturas de las fases ferro-paramagnéticas se obtienen analizando las temperaturas a las cuales $\left|\mathrm{M}_{\mathrm{A}}(\mathrm{T})\right|$ y $\left|\mathrm{M}_{\mathrm{B}}(\mathrm{T})\right|$ son cero. La transición ferro-paramagnética se presenta más rápidamente para el mayor valor de los campos cristalinos anisotrópicos (1.7), y en este caso la subred de espines $\sigma_{j}^{B}$ se desordena más rápido que la subred $A$, fenómeno que se puede entender mediante el máximo que experimenta la curva de $\left|M_{B}(T)\right|$ aproximadamente en $T=1$.
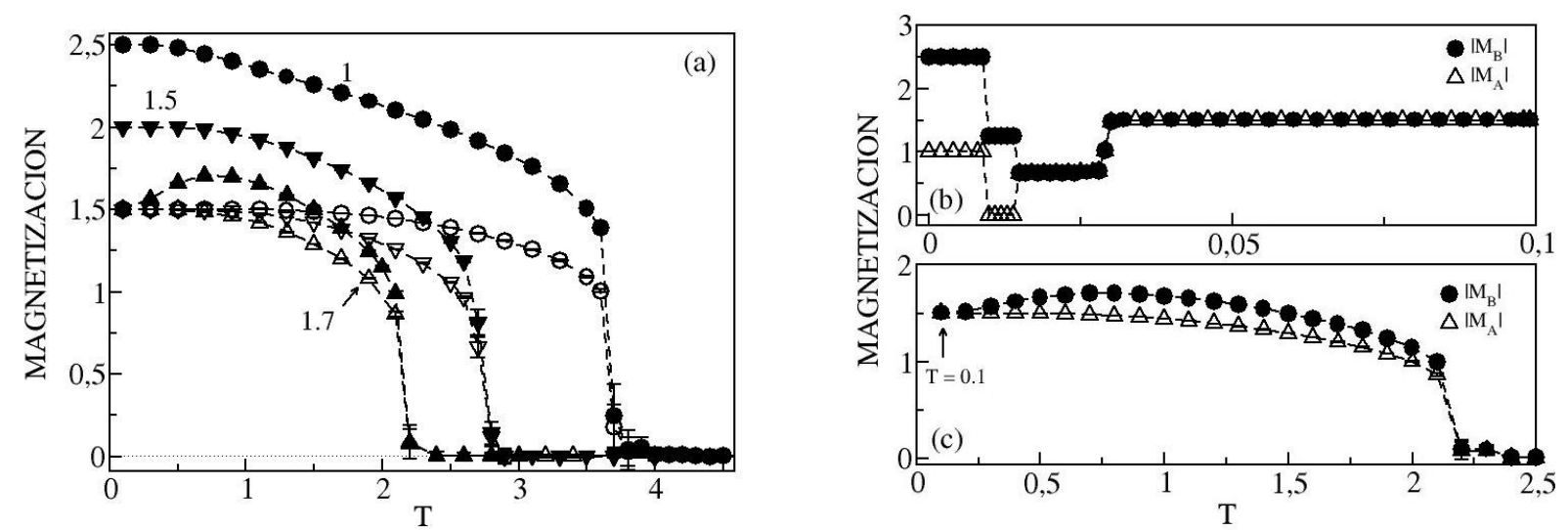

Fig. 3: En (a) $\left|M_{A}(T)\right|$ (símbolos vacíos) y $\left|M_{B}(T)\right|$ (símbolos llenos) para $D_{A}^{\prime}=D_{B}^{\prime}$. Los valores de los campos están sobre las curvas. Los valores de $D_{B}^{\prime}$ para $\left|\mathrm{M}_{A}(T)\right|$ son iguales a los de $\left|\mathrm{M}_{B}(T)\right|$, para el símbolo correspondiente. En (b) $\left|\mathrm{M}_{\mathrm{A}}(\mathrm{T})\right|$ y $\left|\mathrm{M}_{\mathrm{B}}(\mathrm{T})\right|$ en $0 \leq \mathrm{T} \leq 0.1$. En (b) y (c) $D_{A}^{\prime}=D_{B}^{\prime}=$ 1.7.

En las figuras 3(b)-(c) se muestra el comportamiento de las magnetizaciones de las subredes para $D_{A}^{\prime}=D_{B}^{\prime}=1.7$. La gráfica $3(\mathrm{~b})$ exhibe, en las caídas y saltos abruptos de $\left|M_{A}(T)\right|$ y $\left|M_{B}(T)\right|$, posibles transiciones de fase de primer orden, aproximadamente en $0 \leq T \leq 0.03$. También es posible, a estas temperaturas bajas, que el sistema se establezca en cierto estado de metaestabilidad, hasta que las magnetizaciones alcanzan luego sus valores de estados base. En la gráfica 3(c) se muestra el comportamiento de $\left|\mathrm{M}_{A}(T)\right|$ y $\left|\mathrm{M}_{B}(T)\right|$ para $T \geq 0.1$.

Cabe resaltar, en el análisis realizado, que todos los valores seleccionados de los campos cristalinos pertenecen a la región 1 del diagrama de fase de estados base de la figura 1. Para valores grandes de las anisotropías de ión simple $\left(D_{A}^{\prime} \rightarrow \infty, D_{B}^{\prime} \rightarrow \infty\right)$, de acuerdo a la tabla 1, el sistema estaría conformado únicamente por espines $S_{i}^{A}=\mp 3 / 2$ y $\sigma_{j}^{B}=\mp 5 / 2$, y las temperaturas a las cuales se presentan las transiciones de fase ferro-paramagnéticas, serían cada vez menores, e incluso la $T_{C}$ tendería a un valor constante. En consecuencia, el sistema se desordenaría más rápidamente a medida que se incrementa el valor de los campos cristalinos de las subredes.

Los valores seleccionados de $D_{A}^{\prime}$ y $D_{B}^{\prime}$, en las figuras 2 y 3 , para caracterizar el comportamiento termomagnético del sistema, fueron reportados recientemente por Yessoufou y otros autores (Yessoufou et al., 2009), quienes basados en métodos perturbativos, analizaron el modelo utilizando relaciones de recursión exactas sobre una red en forma de árbol de Cayley, con número 
de coordinación $q=4$. Cabe destacar, que en dicha referencia no se reporta el fenómeno de la posible transición de primer orden para $D_{B}^{\prime}=1$, mostrado en la figura 2(a). Análogamente, en la referencia citada, se plantea una transición discontinua próxima a $0.2 \leq \mathrm{T} \leq 0.3$, cuando $D_{A}^{\prime}=D_{B}^{\prime}$ = 1.7; mientras que por nuestro método no perturbativo (Monte Carlo), para este valor de los campos cristalinos, la posible transición se halla aproximadamente en $0 \leq T \leq 0.03$ (Fig. 3(b)).

En la figura 4, se analiza el comportamiento de $\left|\mathrm{M}_{A}(T)\right|$ y $\left|\mathrm{M}_{B}(T)\right|$ para $D_{A}^{\prime}<0$ y $D_{B}^{\prime}>0$. Se aprecia en

las gráficas 4(a)-(c), posibles transiciones de primer orden, y se manifiesta nuevamente mayor ordenamiento de la subred $A$, a partir de su valor de saturación en $T=0$. Para valores grandes de los campos cristalinos, como en las gráficas (b) y (c), la temperatura crítica decrece hasta un valor límite. En la figura 4(c), se acentúa aún más la transición de fase discontinua.
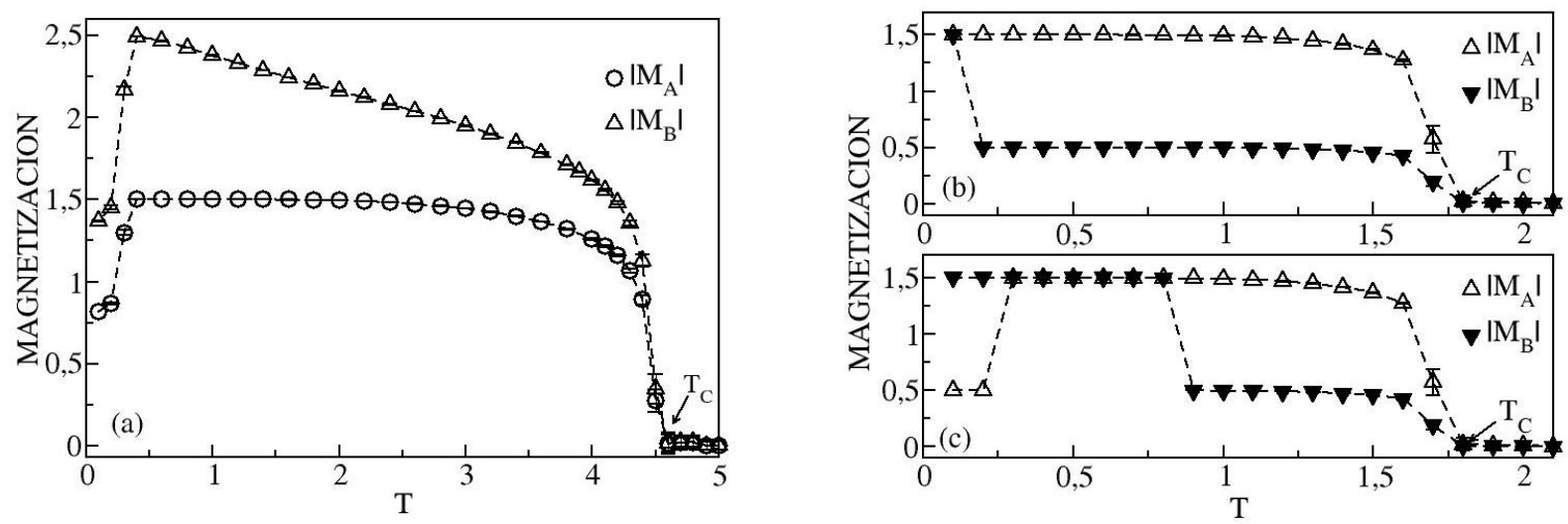

Fig. 4: Comportamiento de las magnetizaciones de las subredes, $\left|\mathrm{M}_{A}(T)\right|$ y $\left|\mathrm{M}_{B}(T)\right|$. En (a), $D_{A}^{\prime}=-$ $1, D_{B}^{\prime}=1$. En (b) $D_{A}^{\prime}=-25, D_{B}^{\prime}=25$. En (c) $D_{A}^{\prime}=-100, D_{B}^{\prime}=100$.

Una forma de estimar las temperaturas críticas del modelo, consiste en estudiar el comportamiento de los calores específicos del sistema para los valores escogidos de los campos $D_{A}^{\prime}$ y $D_{B}^{\prime}$, analizando específicamente la ubicación de sus máximos. En las figuras $5(\mathrm{a})$-(b) se observa que al incrementarse la temperatura, para $T<T_{C}$, el calor específico se incrementa, $y$ para $\mathrm{T}>\mathrm{T}_{\mathrm{C}}$ el incremento de la temperatura implica un decrecimiento del calor específico. En los máximos de las curvas se presentan las transiciones ferro-paramagnéticas, $T=T_{C}$. Estos máximos corresponden a los puntos donde las derivadas de primer orden de la energía interna son discontinuas, y en los cuales ocurren las transiciones de fase de segundo orden en el modelo.
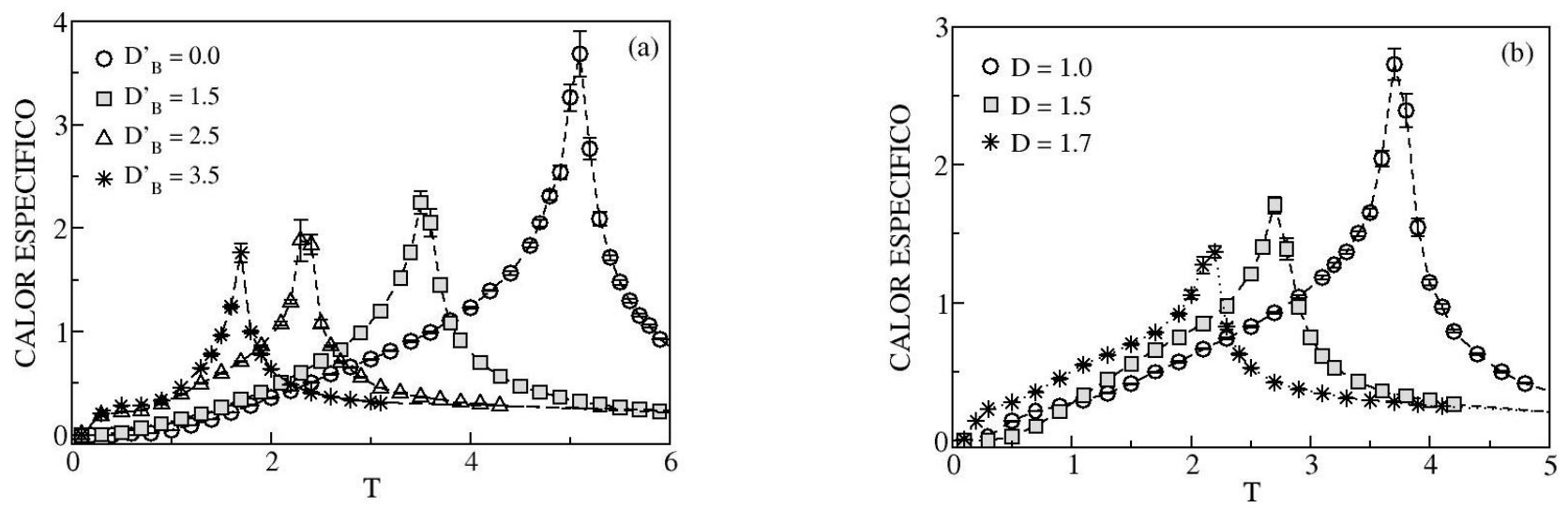

Fig. 5: Comportamiento del calor específico por espín como una función de la temperatura. En (a) se tiene $D_{A}^{\prime}=0$. En (b) $D_{A}^{\prime}=D_{B}^{\prime}=D$.

Cuando $T \rightarrow 0$, en la figura $5(\mathrm{a})$, para $D_{B}^{\prime}=2.5$ y $D_{B}^{\prime}=3.5$, se observan máximos no críticos relacionados con las excitaciones abruptas de espín de la subred $\mathrm{B}$, a causa del efecto de $D_{B}^{\prime}$. 
Cuando los campos cristalinos son nulos, existe un comportamiento normal del calor específico para temperaturas bajas.

El análisis de la influencia de los campos $D_{A}^{\prime}$ y $D_{B}^{\prime}$ sobre la temperatura crítica, se resume en las figuras 6 y 7 . En todos los casos estudiados de la gráfica 6 , la temperatura crítica decrece al incrementarse el módulo del campo cristalino, hasta alcanzar un valor límite que depende de dicho campo. Los resultados indican que el efecto de $D_{B}^{\prime}$ es más crucial que el efecto de $D_{A}^{\prime}$, en el desordenamiento de la red. Se observa, figura $6(\mathrm{a})$, que la región ferromagnética para $D_{B}^{\prime}=0$ (área bajo de la curva), es mayor que el caso cuando $D_{A}^{\prime}=0$. En efecto, la ausencia del campo $D_{A}^{\prime}\left(D_{A}^{\prime}=0\right)$ implica la existencia de un gran número de estados de espín afectados por la dinámica de red. El efecto de los campos con igual magnitud, sobre la temperatura crítica, se observa en la figura $6(\mathrm{~b})$. A medida que se incrementan los campos anisotrópicos, la temperatura crítica decrece hasta alcanzar el valor constante $0.6\left(\mathrm{~T}_{\mathrm{C}}=0.6\right)$ para $|D| / J_{1} \geq 2$, situación reflejada en la figura 3(a).
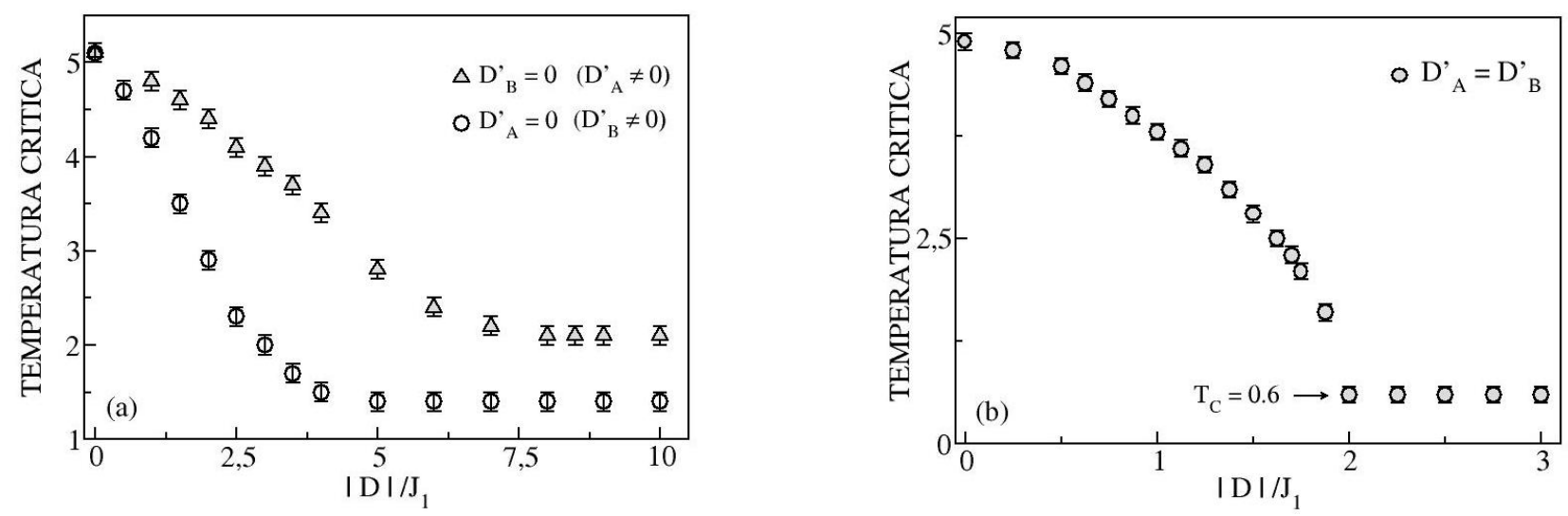

Fig. 6: Comportamiento de la temperatura crítica como una función de los campos cristalinos. En (a) cuando $D_{A}^{\prime}=0, D_{B}^{\prime} \neq 0$, y si $D_{B}^{\prime}=0, D_{A}^{\prime} \neq 0$. En (b) $D_{A}^{\prime}=D_{B}^{\prime}$.

En la gráfica 7 , se considera inicialmente $D_{A}^{\prime}=1$ y varía $D_{B}^{\prime}$, luego se hace el proceso inverso con los mismos valores. La temperatura crítica crece al incrementarse el módulo del campo cristalino, $|D<0|$, hasta alcanzar un valor constante que depende de dicho campo. La temperatura crítica tiene un comportamiento inverso al caso cuando $D_{A}^{\prime}>0$ y $D_{B}^{\prime}>0$ (Fig. 6). Para un mismo valor fijo de $D_{A}^{\prime}$ y $D_{B}^{\prime}$, con $D_{A}^{\prime}<0$ y $D_{B}^{\prime}<0$, el campo $D_{A}^{\prime}$ genera más desorden en el sistema que $D_{B}^{\prime}$. Se nota en la gráfica 7 que la región paramagnética, debida al efecto de $D_{A}^{\prime}$, es mayor que la ocasionada por la influencia de $D_{B}^{\prime}$ (región por encima de la curva).

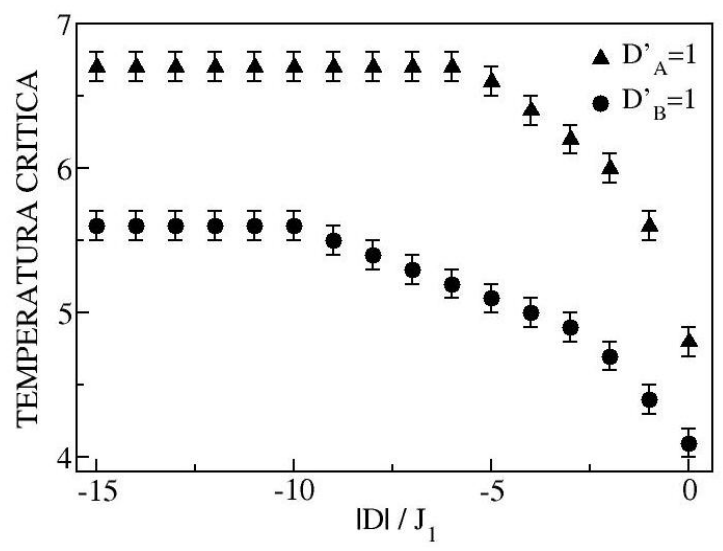

Fig. 7: Comportamiento de la temperatura crítica como una función de los campos cristalinos.

Cuando $D_{A}^{\prime}=1, D_{B}^{\prime} \leq 0$ (círculos), y si $D_{B}^{\prime}=1, D_{A}^{\prime} \leq 0$ (triángulos).

La anisotropía magnética se cuantifica por la energía requerida para orientar la magnetización de saturación desde su eje fácil hacia su eje difícil. Los resultados físicos de esta investigación se justifican en el efecto que produce la anisotropía magnética, generada por los campos de las 
subredes, sobre la temperatura crítica del sistema. También, como a través de modelos mixtos de Ising se pueden explicar cualitativamente diversos aspectos del comportamiento magnético de algunos compuestos moleculares, tal como la independencia de la temperatura crítica en la concentración de los diferentes espines.

\section{CONCLUSIONES}

Se estudia el comportamiento termomagnético del sistema de Ising mixto ferromagnético de espines $S_{i}^{A}=3 / 2$ y $\sigma_{j}^{B}=5 / 2$, sobre una red bidimensional cuadrada, mediante simulaciones de Monte Carlo. Se analiza el comportamiento a temperatura finita de las magnetizaciones de las subredes, el calor específico y $\mathrm{T}_{\mathrm{C}}$, con relación a los campos cristalinos $D_{A}^{\prime}$ y $D_{B}^{\prime}$. Se hallan posibles transiciones de primer orden, para $\left|\mathrm{M}_{A}(\mathrm{~T})\right|$ y $\left|\mathrm{M}_{B}(\mathrm{~T})\right|$, cuando $D_{A}^{\prime}=D_{B}^{\prime}=1.7, D_{A}^{\prime}=0$ y $D_{B}^{\prime}$ $=1, D_{A}^{\prime}=-1$ y $D_{B}^{\prime}=1, D_{A}^{\prime}=-100$ y $D_{B}^{\prime}=100$. Para $D_{A}^{\prime}=0$ y $D_{B}^{\prime}>0$, la $T_{C}$ decrece hasta alcanzar un valor límite, de igual forma ocurre cuando $D_{B}^{\prime}=0$ y $D_{A}^{\prime}>0$. Cuando se fija un valor positivo de cualquiera de los campos $(D>0)$, al incrementarse el módulo del otro, $|D<0|$, la $T_{C}$ se incrementa hasta un valor constante. Para $D_{A}^{\prime}>0$ y $D_{B}^{\prime}>0$, el efecto del campo $D_{B}^{\prime}$ es más influyente en el desorden del sistema, mientras que para $D_{A}^{\prime}<0$ y $D_{B}^{\prime}<0$ el efecto del campo $D_{A}^{\prime}$ perturba más el sistema. Con este trabajo se verifican las características cualitativas de los resultados de la referencia (Yessoufou et al., 2009), en lo que respecta al comportamiento de $\left|\mathrm{M}_{\mathrm{A}}(\mathrm{T})\right|,\left|\mathrm{M}_{\mathrm{B}}(\mathrm{T})\right|$ y $\mathrm{T}_{\mathrm{C}}$ cuando el número de coordinación es $q=4$, y $D_{A}^{\prime}>0$ y $D_{B}^{\prime}>0$. La técnica no perturbativa (Monte Carlo) usada en este estudio implica que los resultados sean más precisos, ya que la aproximación usada en la referencia citada tiende a sobreestimar los valores de la $T_{C}$.

\section{REFERENCIAS}

Albayrak, E. y A. Yigit, The phase diagrams of de mixed spin-3/2 and spin-5/2 Ising system on the Bethe lattice, Physica status solidi b: 244, 748-758 (2007).

Belmamoun, Y., H. Ez-Zahraouy y M. Kerouad, Monte Carlo study of the spin-1/2 Ising system with antiferromagnetic surface on a ferromagnetic material, Journal of Manetism and Magnetic Materials: 321, 635-639 (2009).

Benayad, N. y M. Ghliyem, Mixed spin Ising model with four-spin interaction and random crystal field, Physica B: 407, 6-13 (2012).

Blundell, S. J. y F. L. Pratt, Organic and molecular magnets, Journal of Physics: Condensed Matter: 16, 771-828 (2004).

Buendía, G. y J. Villarroel, Compensation temperatures of mixed ferro-ferrimagnetic ternary alloys, Journal of Magnetism and Magnetic Materials: 310, E495-E497 (2007).

De La Espriella, N. y G. Buendía, Ground state phase diagrams for the mixed Ising 3/2 and 5/2 spin model, Physica A: 389, 2775-2732 (2010).

De La Espriella, N. y G. Buendía, Magnetic behavior of a mixed Ising 3/2 and 5/2 spin model, Journal of Physics: Condensed Matter: 23, 176003-176009 (2011).

De La Espriella, N., G. Casiano y C. Ortega, Propiedades magnéticas del sistema ferrimagnético de Ising mixto de espines $S=3 / 2$ y $\sigma=5 / 2$, Información Tecnológica: 23 (2) en prensa (2012a).

De La Espriella, N., G. Casiano y C. Ortega, Estudio Monte Carlo de un ferrimagneto de Ising mixto con diferentes anisotropías, Información Tecnológica: 23 (2) en prensa (2012b).

Dely, J., A. Bobak y M. Zukovic, Compensation temperatures and magnetic susceptibility of a mixed ferro-ferrimagnetic ternary alloy, Physics Letters A: 373, 3197-3200 (2009).

Deviren B., M. Keskin y O. Canko, Kinetic Ising model in a time-dependent oscillating external magnetic field: Effective-field theory, Journal of Magnetism and Magnetic Materials: 321, 458-466 (2009). 
Deviren, B. y M. Keskin, Dynamic phase transitions and compensation temperatures in a mixed spin3/2 and spin-5/2 Ising system, Journal of Statistical Physics: 140, 934-947 (2010).

Deviren, B., S. Akbudak y M. Keskin, Mixed spin-1 and spin-3/2 Ising system with two alternative layers of a honeycomb lattice within the effective-field theory, Solid State Communications: 151, 193-198 (2011).

Deviren, B., M. Ertas y M. Keskin, The effective-field theory studies of critical phenomena in a mixed spin-1 and spin-2 Ising model on honeycomb and square lattices, Physica A: 389, 2036-2047 (2010).

Gatteshi, D. y R. Sessoli, Molecular nanomagnets: the first 10 years, Journal of magnetism and magnetic materials: 272-276, 1030-1036 (2004).

Hurtado, N. y G. Buendía, Efecto del campo cristalino en un ferrimagneto mixto de Ising tridimensional, Revista Mexicana de Física: 49(3), 183-185 (2003).

Keskin, M., O. Canko y S. Guldal, Phase diagrams of a nonequilibrium mixed spin-1/2 and spin-2 Ising ferrimagnetic system under a time-dependent oscillating magnetic field, Physics Letters A: 374, 1-7 (2009).

Keskin, M., e Y. Polat, Phase diagrams of a nonequilibrium mixed spin-3/2 and spin-2 Ising system in an oscillating magnetic field, Journal of Magnetism and Magnetic Materials: 321, 3905-3912 (2009).

Luo, S. J., Z. D. He y Q. L. Jie, A Monte Carlo study of the thermodynamic properties of a quasi-onedimensional organic polymer ferromagnet, Journal of Magnetism and Magnetic Materials: 321, 33963401 (2009).

Mohamad, H., Spin compensation temperatures induced by longitudinal fields in a mixed spin-3/2 and spin-5/2 Ising ferrimagnet, Journal of Magnetism and Magnetic Materials: 323, 61-66 (2011).

Nakamura, Y. y J. W. Tucker, Monte Carlo study of a mixed spin-1 and spin-3/2 Ising ferromagnet, IEEE Transactions on Magnetics: 38, 2406-2408 (2002).

Newman, M. E. J. y G. T. Barkema, Monte Carlo Methods in Statistical Physics, $4^{\text {a }}$ edición, 68-70. Oxford University Press, New York, United States, (2006).

Ohkoshi, S. y K. Hashimoto, New Magnetic Functionalities Presented by Prussian Blue Analogues, Electrochemical Society Interface: 11, 34-38 (2002).

Selke, W. y J. Oitmaa, Monte Carlo study of mixed-spin $S=(1 / 2,1)$ Ising ferrimagnets, Journal of Physics: Condensed Matter: 22, 076004 (2010).

Yessoufou, R. A., S. H. Amoussa y F. Hontinfinde, Magnetic properties of the mixed spin-5/2 and spin$3 / 2$ Blume-Capel Ising system on the two-fold Cayley tree, Central European Journal of Physics: 7(3), 555-567 (2009).

Zaim, A., M. Kerouad e Y. E. Amraoui, Magnetic properties of a ferrimagnetic core/shell nanocube Ising model: A Monte Carlo simulation study, Journal of Magnetism and Magnetic Materials: 321, 1077-1083 (2009a).

Zaim, A., M. Kerouad e Y. Belmamoun, Monte Carlo study of a mixed spin-1/2 and spin-1 Blume-Capel ferrimagnetic model with four-spin interaction, Physica B: 404, 2280-2284 (2009b).

Zaim, A. y M. Kerouad, Monte Carlo simulation of the compensation and critical behaviors of a ferrimagnetic core/shell nanoparticle Ising model, Physica A: 389, 3435-3442 (2010).

Zhang, Q., G. Wei e Y. Gu, The study of the phase diagram and internal energy of the mixed spin-3/2 and spin-5/2 ferrimagnetic Ising system with interlayer coupling by effective-field theory; a simple approach of calculating internal energy, Physica status solidi b: 242 (4), 924-932 (2005).

Zukovic, M. y A. Bobak, Phase diagram of a mixed spin-1 and spin-3/2 Ising ferrimagnet, Physica A: 389, 5402-5407 (2010). 